\title{
The effect of marine $n-3$ polyunsaturated fatty acids on cardiac autonomic and hemodynamic function in patients with psoriatic arthritis: a randomised, double- blind, placebo-controlled trial
}

Salome Kristensen ${ }^{1 *}$ (D), Erik Berg Schmidt ${ }^{2}$, Annette Schlemmer ${ }^{1}$, Claus Rasmussen³, Esther Lindgreen', Martin Berg Johansen ${ }^{4}$ and Jeppe Hagstrup Christensen ${ }^{5}$

\begin{abstract}
Background: The aim of this study was to investigate the effect of marine $n-3$ polyunsaturated fatty acids (PUFA) on cardiac autonomic function and vascular function in patients with psoriatic arthritis.

Methods: The study was conducted as a randomized, double-blind, placebo-controlled trial, where 145 patients with psoriatic arthritis were supplemented with $3 \mathrm{~g}$ of $\mathrm{n}-3$ PUFA or olive oil (control) daily for 24 weeks. Blood pressure, heart rate, heart rate variability (HRV), central blood pressure, pulse wave velocity (PWV) and fatty acid composition of granulocytes, were determined at baseline and after supplementation.

Results: At baseline we found a significant difference in the mean of all normal RR intervals (inverse of heart rate, vary from beat to beat) when comparing subjects with the highest vs the lowest fish intake $(p=0.03)$. After supplementation for 24 weeks there was a trend towards an increase in $\mathrm{RR}(p=0.13)$ and decrease in heart rate $(p=0.12)$ comparing the $n-3$ PUFA group with the control group. However, per-protocol analysis showed significantly increased RR $(p=0.01)$ and lowered heart rate $(p=0.01)$ in the n-3 PUFA supplemented patients compared with controls. Blood pressure, PW and Central blood pressure did not change after supplementation with n-3 PUFA. Adjustment for disease activity and conventional cardiovascular risk factors did not change the results.
\end{abstract}

Conclusions: Marine n-3 PUFA increased RR intervals in patients with psoriatic arthritis which may suggest a protective effect of n-3 PUFA against cardiovascular disease in this population.

Trial registration: Clinicaltrials.gov Identifier: NCT01818804

Keywords: Psoriatic arthritis, n-3 PUFA, Heart rate variability, Cardiac autonomic function, Pulse wave velocity, Arterial stiffness

\footnotetext{
* Correspondence: sakr@rn.dk

1 Department of Rheumatology, Aalborg University Hospital, Reberbansgade

14, 9000 Aalborg, Denmark

Full list of author information is available at the end of the article
} 


\section{Background}

Psoriatic arthritis (PsA) is a chronic inflammatory joint disease occurring in 6-39\% of patients with psoriasis and with a prevalence in the general population of approximately $0.2 \%$ [1-3].

Patients with inflammatory diseases such as rheumatoid arthritis and systemic lupus erythematosus are at an increased risk of cardiovascular disease (CVD) [4] and recent observational studies indicate that PsA patients also have an increased cardiovascular mortality and morbidity $[5,6]$. Accelerated atherosclerosis due to inflammation, and autonomic dysfunction can both play a role in the pathogenesis of CVD in patients with PsA in addition to conventional risk factors for CVD such as smoking, hypertension, hypercholesterolemia and diabetes mellitus [7].

Several studies have indicated that chronic inflammation may impair autonomic cardiac regulation leading to a decrease in heart rate variability (HRV) [8, 9]. A low HRV has been identified as an independent predictor of coronary heart disease [10], as well as malignant ventricular arrhythmias and sudden cardiac death [11-15]. Also, arterial stiffness has been recognised as an independent predictor of CVD $[16,17]$. Studies using noninvasive methods such as pulse wave velocity (PWV) for evaluation of CVD risk have revealed increased arterial stiffness in patients with PsA [18-20].

A beneficial effect of marine $n-3$ polyunsaturated fatty acids (PUFA) on CVD has been suggested from several epidemiological studies, experimental data and clinical trials [21]. Interestingly, the major marine n-3 PUFA, eicosapentaenoic acid (EPA; 20:5n-3) and docosahexaenoic acid (DHA; 22:6n-3), have been shown to have antiinflammatory effects [22] and beneficial effects on blood pressure (BP) and cardiac autonomic function [23, 24].

The present study aimed to examine whether supplementation with a moderate to high ( $3 \mathrm{~g}$ ) daily dose of marine n-3 PUFA for 24 weeks had a beneficial effect on cardiac autonomic and hemodynamic function represented by BP, heart rate (HR), HRV, PWV and central BP in patients with PsA.

\section{Methods}

\section{Study design}

The study was designed as a randomized, double-blind, placebo-controlled trial. The patients were randomly assigned in blocks of five by a computer-generated block sequence. For 24 weeks patients were assigned to daily intake of six capsules containing either $3 \mathrm{~g}$ of $\mathrm{n}-3$ PUFA (50\% EPA and $50 \%$ DHA) or $3 \mathrm{~g}$ of olive oil (approximately $80 \%$ of oleic acid and $20 \%$ linoleic acid). Investigators, patients and research staff were blinded to the supplementation codes. Patients were asked to maintain their usual diet during the entire study. The study was conducted in a 2 year period between 2013 and 2015 . Clinical assessment, blood samples, HRV and PWV were carried out at baseline and after 24 weeks. The study was conducted in accordance with the Declaration of Helsinki and registered at ClinicalTrials.gov (NCT01818804). Furthermore the study was monitored by Good Clinical Practice (GCP) inspectors and the GCP ethical and scientific quality requirements were followed.

\section{Subjects}

Patients with PsA defined by Classification criteria for psoriatic arthritis (CASPAR) [25] were enrolled from the Department of Rheumatology, Aalborg University Hospital and Department of Rheumatology, Vendsyssel Hospital, in Denmark. The inclusion criteria were PsA in adults above 18 years of age with any disease activity while exclusion criteria were documented known cardiac arrhythmias, treatment with biological drugs, or treatment with oral corticosteroids. Patients using fish oil supplements $(n=2)$ underwent a wash-out period of at least 8 weeks before inclusion.

Compliance was assessed by counting capsules during the last visit. Patients were defined as non-compliant if missing $>15 \%$ of capsules and these patients were not included in the per-protocol analysis.

All participants gave their written informed consent and the regional ethics committee of Northern Region DK, approved the study (reference number N20120076).

\section{Clinical assessment}

At baseline, duration of PsA, medical history, smoking habits and diets were obtained. Medical history of diabetes mellitus, hypertension and dyslipidemia was assessed and was defined as present if the patient received dietary or medical therapy for the condition. A food questionnaire was used to assess patients' fish consumption at lunch and dinner. A score for fish intake was given according to the following: never eating fish = 1 ; eating fish once a month $=2$; eating fish two to three times a month $=3$; eating fish once a week $=4$; eating fish two to three times a week $=5$; and eating fish at least once daily $=6$.

At both visits conventional cardiovascular risk factors such as smoking habits, BP, body mass index (BMI) and waist to hip ratio (WHR) were assessed. Additionally, a clinical evaluation was performed, consisting of 68 tender joint count, 66 swollen joint count, disease activity score (DAS66/68) and psoriatic skin area involvement (PASI).

\section{Blood samples}

Blood samples were taken non-fasting for assessment of fatty acid composition of granulocytes and routine laboratory evaluation including plasma levels of C-reactive 
protein (CRP). Granulocytes were isolated from whole blood and their fatty acid composition were determined by gas chromatography with a Chrompack CP-9002 gas chromatograph (Varian, Middelberg, The Netherlands) and expressed as weight percent (wt \%) of total fatty acids. The following fatty acids were evaluated in the further analyses: eicosapentaenoic acid (EPA; 20:5n-3), docosahexaenoic acid (DHA; 22:6n-3), docosapentaenoic acid (DPA; 22:5n-3), arachidonic acid (AA; 20:4n-6), oleic acid (18:1n-9), linoleic acid (LA; 18:2n-6), palmitic acid (16:0) and stearic acid (18:0).

\section{HRV}

Five min. HRV recordings were obtained with SphygmoCor Technology (SphygmoCor, Software version 8.2; AtCor Medical, Sydney, NSW, Australia) in each patient. HRV was recorded according to current recommendations [26] with measurements obtained in the morning hours after resting for $15 \mathrm{~min}$ in a room with a constant temperature of $20^{\circ} \mathrm{C}$. Patients were instructed not to smoke and avoid alcohol and caffeine-containing beverages within $12 \mathrm{~h}$ prior to investigation. A trained technician blinded to the type of supplement performed these analyses. The patients were in a supine position (resting) for $10 \mathrm{~min}$, breathing spontaneously without talking. HRV were analysed in the timedomain and the following variables were obtained:

- HR: heart rate

- RR: mean of all normal RR intervals during the 5 min recording (inverse of heart rate, vary from beat to beat)

- SDNN: standard deviation of all normal RR intervals in the 5 min recording

- SDNNindex: mean of the standard deviation of all the normal RR intervals

- pNN50: percentage of successive RR-interval differences $>50 \mathrm{~ms}$

- RMSSD: square root of the mean of the sum of the squares of differences between adjacent intervals

\section{PWV}

PWV and pulse wave analysis were performed noninvasively with the Sphygmocor system (AtCor Medical, Sydney, NSW, Australia), as described previously [27] and according to international recommendations [28]. All measurements were made in duplicate by a single trained operator and the mean of the two values was used in the analysis. Carotid-radial and carotid-femoral PVW were measured using arterial tonometry. The measurements were obtained under the same conditions as described under HRV. The patients were in a supine position and all measurements were performed on the right side extremities. The surface distance was measured with a tape measure as a straight line from the suprasternal notch to the carotid location (proximal pulse) and subtracted the distance from the suprasternal notch to the radial or femoral location (distal pulse) [29,30]. The pressure wave transit time was determined as the time between the Rwave of the ECG and the proximal pulse subtracted from the time between the R-wave of the ECG and the distal pulse. PWV was subsequently calculated by dividing the surface distance by the pressure wave transit time.

The central BP was estimated using the SphygmoCor ${ }^{\circ}$ device. After $10 \mathrm{~min}$ of rest in the supine position, brachial BP was measured three times at 2-min intervals on the left arm with an automatic Microlife ${ }^{\circ}$ device, and the last measurement was taken as representative of brachial artery BP. Hereafter, radial artery pressure waveforms of the right arm were sampled. Using the validated generalized transfer function, central BP was estimated using brachial systolic and diastolic BP [31, 32]. Aortic augmentation index (AIx) was standardized to a HR of 75 beat per minute to minimize the effect of HR.

\section{Statistical Analysis}

All statistical analyses were performed using Stata: Release 13 (StataCorp LP, TX, US). HRV measurements were primary outcome and PWV measurements secondary outcome.

We hypothesized that intervention with n-3 PUFA would increase RR. Based on previous literature [33, 34] and to achieve $\alpha=0.05$ and $1-\beta=0.80$ we needed a sample size with 63 subjects in each group.

The score for fish intake at baseline was grouped according to tertiles. The content of DHA and EPA in granulocytes were also grouped according to tertiles.

The difference in the continuous outcomes between baseline and 24 weeks after randomization was compared between the two treatment groups in a one-way analysis of variance (ANOVA). Equality of variances between the treatment groups was assessed using Bartlett's test. Due to the potential for confounding after the random treatment assignment an analysis of covariance (ANCOVA) was performed. Prior to this analysis a check for collinearity between the covariates was performed and the model was modified accordingly. In this model equality of variances was assessed using Levene's test. All analyses were performed both as intention to treat and per-protocol (patients who completed the entire clinical trial according to the protocol and consumed $>85 \%$ of the assigned supplement) analyses. The ANCOVA analyses were controlled for age, sex, smoking status, presence or absence of diabetes mellitus, hypertension and hypercholesterolemia, use of nonsteroidal antiinflammatory drugs (NSAID) and DAS66/68.

Differences were considered significant with a $p$-value of $<0.05$ (two-tailed). 


\section{Results}

A total of 145 patients were enrolled and 133 patients (92\%) completed the study. Seven patients in the control arm and five patients in the n-3 PUFA arm withdrew from the study before last visit and five patients were excluded because of insufficient HRV recordings at baseline. Ten patients in the n-3 PUFA group and four in the control group were defined as noncompliant according to the number of capsules returned and were not included in the per-protocol analysis. Figure 1 shows the study flow diagram.

The n-3 PUFA and the control groups were comparable regarding baseline characteristics, apart from number of current smokers and number of patients with known hypercholesterolemia (Table 1). Thus, adjustments were made for these to parameters in the final analysis. Patients excluded from final analysis did not differ from patients eligible for final analysis in regard to baseline characteristics (data not shown). Nine participants in the n-3 PUFA supplemented group and six participants in the control group reported mild gastrointestinal adverse effect. In 13 of these participants the side effects was avoided by changing the intake of capsules from six capsules once daily to two capsules three times daily. The remaining two participants were in the placebo group and dropped out because of nausea and mild diarrhea.

\section{Baseline analyses}

Patients with the highest fish intake had a significantly higher RR than patients with the lowest intake $(p=0.03)$. Also, patients in the tertile with the highest content of DHA in granulocytes had the highest RR $(p=0.04)$ whereas the content of EPA in granulocytes was not associated with RR. The associations between dietary fish intake and RR seemed to be dose dependent (Figs. 2 and $3)$. There were no association between DAS66/68 and $\mathrm{RR}$ at baseline.

\section{Intervention analyses}

The group randomized to $n-3$ PUFA had a significant increase in total content of n-3 PUFA (DHA + EPA + DPA), DHA and EPA in granulocytes from baseline to study end compared to the control group. There were

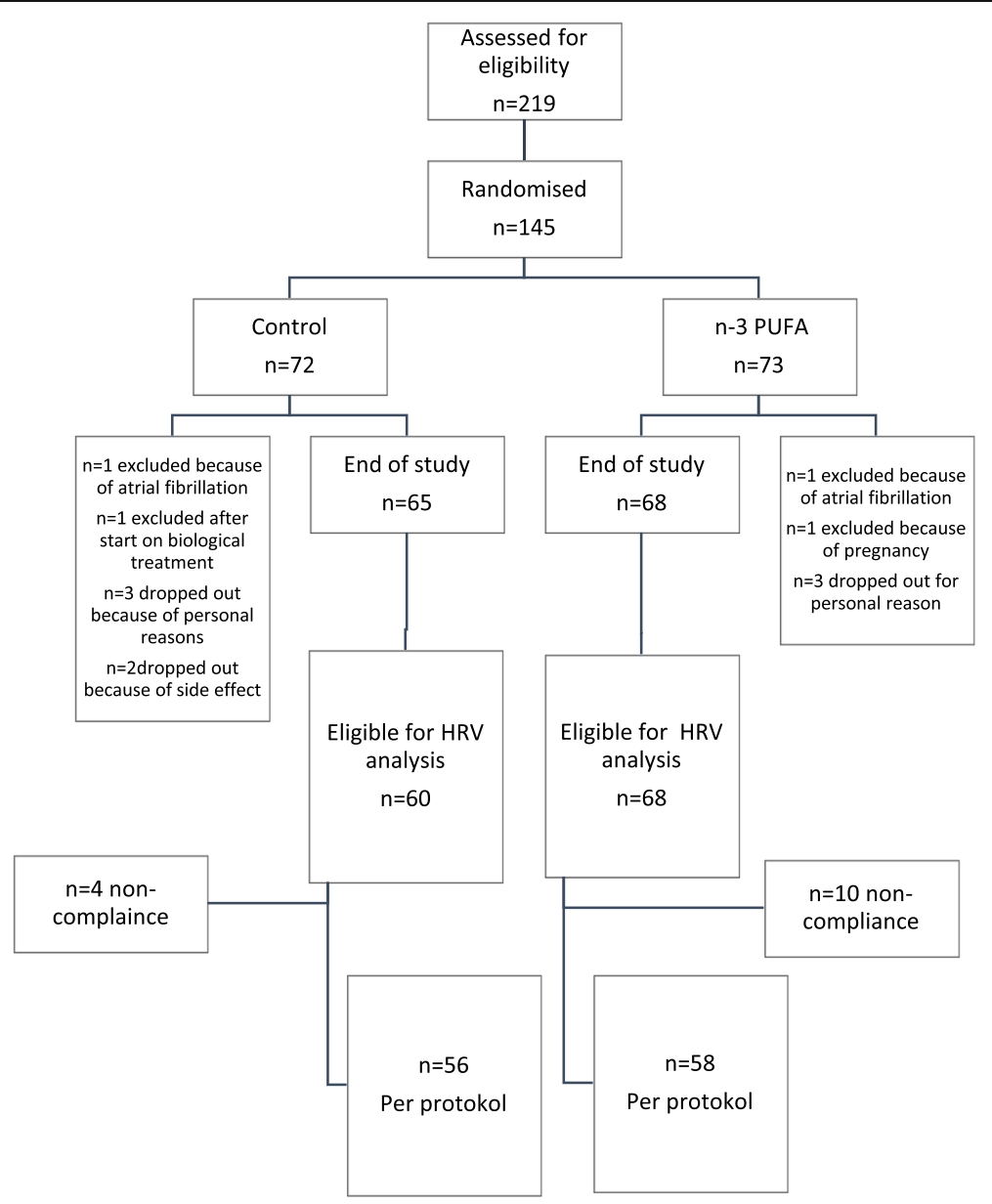

Fig. 1 Flow diagram of the study participants 
Table 1 Baseline demographics, biochemical and hemodynamic characteristics of the 143 patients with psoriatic arthritis

\begin{tabular}{|c|c|c|c|c|}
\hline & & $\begin{array}{l}n-3 \text { PUFA } \\
(N=72)\end{array}$ & $\begin{array}{l}\text { Control } \\
(N=71)\end{array}$ & $\begin{array}{l}\text { Total } \\
(N=143)\end{array}$ \\
\hline \multirow[t]{13}{*}{ Covariates } & Age & & & \\
\hline & Mean age & $53.2(11.4)$ & $50.7(11.5)$ & $52.0(11.5)$ \\
\hline & Sex & & & \\
\hline & - Female & $40(55.6)$ & $43(60.6)$ & $83(58.0)$ \\
\hline & - Male & $32(44.4)$ & $28(39.4)$ & $60(42.0)$ \\
\hline & Smoking & & & \\
\hline & - Non-smoker & $36(50.0)$ & $41(57.7)$ & $77(53.8)$ \\
\hline & - Former smoker & $27(37.5)$ & $14(19.7)$ & $41(28.7)$ \\
\hline & - Current smoker & $9(12.5)$ & $16(22.5)$ & $25(17.5)$ \\
\hline & $\begin{array}{l}\text { Documented coronary } \\
\text { heart disease }\end{array}$ & $4(5.6)$ & $4(5.6)$ & $8(5.6)$ \\
\hline & Hypertension & $21(29.2)$ & $20(28.2)$ & $41(28.7)$ \\
\hline & Hypercholesterolemia & $18(25.0)$ & $11(15.5)$ & $29(20.3)$ \\
\hline & NSAID use & $40(55.6)$ & $32(45.1)$ & $72(50.3)$ \\
\hline \multirow[t]{8}{*}{ Outcomes } & Systolic BP, mmHg & $138(18)$ & $134(19)$ & $136(19)$ \\
\hline & Diastolic BP, mmHg & $83(11)$ & $82(12)$ & $82(12)$ \\
\hline & Hip and waste ratio & $1.1(0.1)$ & $1.2(0.1)$ & $1.1(0.1)$ \\
\hline & Body Mass Index & $28.6(5.7)$ & $28.0(5.0)$ & $28.3(5.4)$ \\
\hline & Disease Activity Score & $2.5(0.9)$ & $2.7(0.9)$ & $2.6(0.9)$ \\
\hline & CRP mg/l & $4.6(4.2)$ & $6.1(7.7)$ & $5.3(6.2)$ \\
\hline & PASI & $2.2(3.0)$ & $2.3(4.0)$ & $2.3(3.5)$ \\
\hline & Total cholesterol, mmol/l & $5.0(1.0)$ & $4.8(0.8)$ & $4.9(0.9)$ \\
\hline
\end{tabular}

Data are given as mean (sd) or $n$ (\%) as appropriate $N$ non-missing values, $B P$ Blood pressure

also a significant decrease in the granulocyte content of AA and LA in the n-3 PUFA supplemented group compared to control group (Table 2).

\section{Intention to treat analysis}

After supplementation for 24 weeks there was a trend towards an increase in RR $(p=0.06)$ and a decrease in HR $(p=0.12)$ comparing the $n-3$ PUFA group with the control group (Table 3).

There were no significant change in BP, PWV or central $\mathrm{BP}$ in the $\mathrm{n}-3$ PUFA supplemented group or between the n-3 PUFA and control group. Adjustment for conventional CVD risk factors and DAS66/68 did not affect the results.

DAS66/68 and CRP were not associated with HRV or PWV.

\section{Per-protocol analysis}

Analyses of outcomes after 24 weeks revealed a significant increase in RR and decrease in HR in the fish oil supplemented group and there was a significant difference in changes in RR $(p=0.03)$ and HR (0.02) between the n-3 PUFA and control group (Table 4). In contrast, there were no significant change in BP, PWV or central BPs in the n-3 PUFA supplemented group or between the n-3 PUFA and control group. Adjustment for conventional CVD risk factors and DAS66/68 did not change the results (Table 5).

\section{Discussion}

With 145 participants and only $8 \%$ not completing, this study is the largest investigation of the effect of marine n-3 PUFA on cardiovascular function in patients with PsA. We found that supplementation with $3 \mathrm{~g}$ n-3 PUFA daily for 24 weeks had a beneficial effect on autonomic control of the heart in PsA patients by decreasing heart rate and increasing RR interval.

At baseline we found a significantly higher RR in the patients with the highest fish intake. Baseline analysis also showed that the content of DHA in granulocytes was positively associated with RR. These associations seemed to be dose dependent and the results suggest beneficial effect of dietary fish consumption on the cardiac autonomic tone in patients with PsA.

After intervention for 24 weeks there was a significant shift in the PUFA composition in the active group with an increase in the granulocyte content of n-3 PUFA and a decrease in content of AA and LA. In the intention to treat analysis there was a trend towards increased $R R$ and thereby a reciprocal lowering of HR. However, the per-protocol analysis revealed a significant increase in $R R$ and decrease in HR in the n-3 PUFA supplemented group.

HRV is considered a useful and reliable measurement of cardiac autonomic tone [35]. A depressed HRV indicates an increased cardiovascular risk in the general population [11] and in patients with known CVD [36]. Evidence also suggests that HRV improves with fish consumption and intervention with n-3 PUFA [37]. The positive association between fish intake and RR at baseline and the increase in RR after intervention with $n-3$ PUFA found in this study of patients with PsA is in line with previous studies of other high-risk patients and healthy subjects [38]. The effect of n-3 PUFA on HR in our study is also consistent with previous data showing that $n-3$ PUFA reduces resting HR $[39,40]$, an important risk marker for cardiovascular disease [41]. As a surrogate marker for cardiac autonomic tone, HRV can indicate changes mediated by n-3 PUFA at the level of cardiac efferent stimuli [42, 43]. Interestingly, in our study baseline content of DHA but not EPA in granulocytes was positively associated with $R R$, which is consistent with findings of previous studies [44, 45]. DHA is most abundant in the heart, brain and nervous system membrane lipids [46] and therefore might be more influential regarding effects on cardiac autonomic function. 


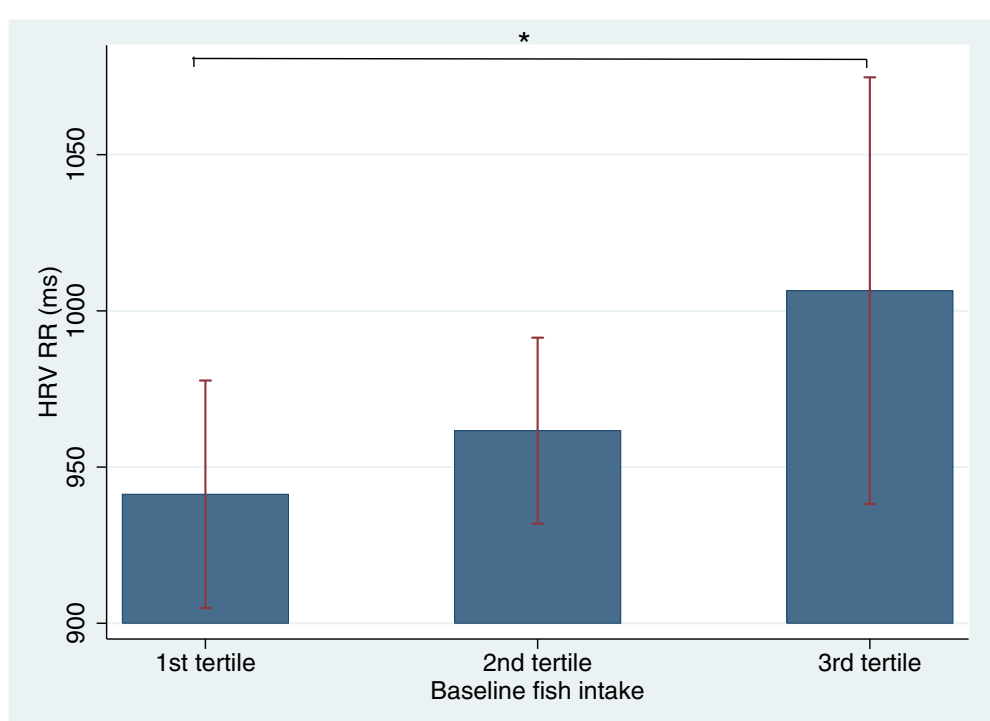

Fig. 2 The relation between baseline RR (ms) and fish intake presented in tertiles. Footnote: RR: Mean of all normal RR-intervals in HRV recording; *: Significant difference in RR between the lower and the upper tertile

Interactions between the autonomic nervous system and the immune system have been reviewed recently and direct autonomic innervation and non-synaptic communication with lymphoid organs has been shown [47]. Abnormalities in the autonomic nervous system as well as cardiovascular autonomic dysfunction has been reported in patients with inflammatory rheumatic diseases [48]. A few studies have investigated HRV in PsA and demonstrated attenuated HRV. In a study with 38 patients with PsA and 25 healthy controls using 5-min
HRV Gaydukova et al. [49] found that SDNN was $65.1 \mathrm{~ms}$ and pNN50 12.9\% in the PsA patients whereas in healthy controls SDNN and pNN50 were higher (83.2 ms and 20.6\%, respectively). Proietti et al. [50] also used short-term HRV in 26 patients with psoriasis and 27 healthy controls and found a significant difference in RMSDD between patients with psoriasis $(39.4 \mathrm{~ms})$ and the controls $(53.2 \mathrm{~ms})$. Our results revealed a baseline SDNN of $50 \mathrm{~ms}$, pNN50 of 13\%, and RMSSD of $38 \mathrm{~ms}$, supporting an attenuation of HRV in patients with PsA.

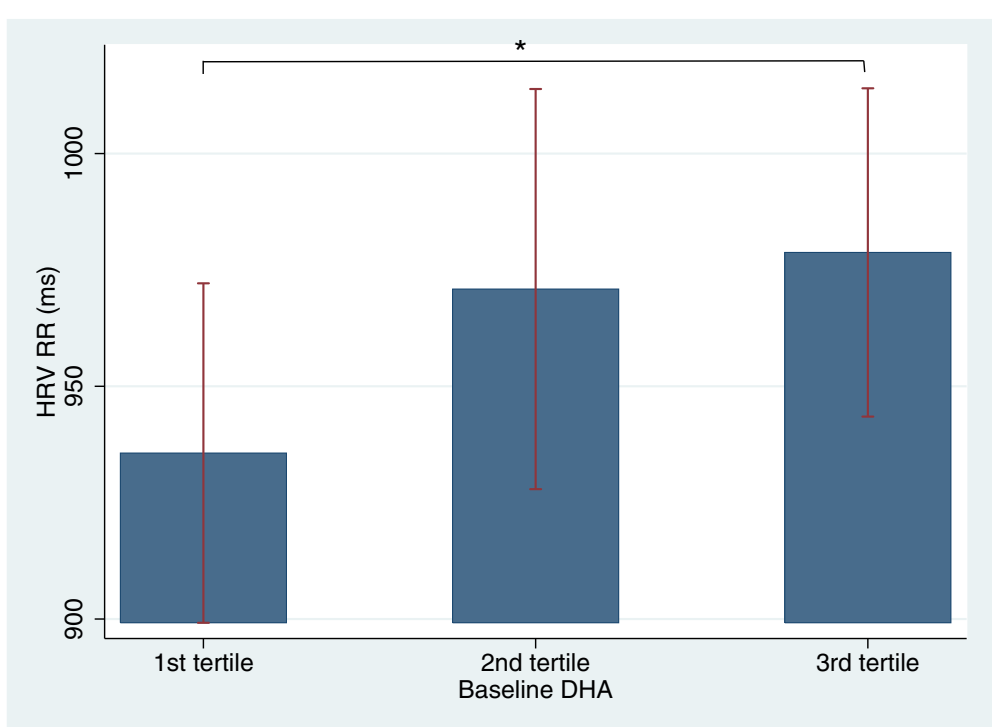

Fig. 3 The relation between baseline RR (ms) and content of DHA in granulocytes presented in tertiles. Footnote: HRV: Heart Rate Variability; RR: Mean of all normal RR-intervals in HRV recording; DHA: docosahexaenoic acid; *: Significant difference in RR between the lower and the upper tertile 
Table 2 Content of fatty acids in granulocytes presented as means with 95\% confidence intervals at baseline and after 24 weeks of supplementation for both groups

\begin{tabular}{|c|c|c|c|c|c|c|c|}
\hline & \multicolumn{3}{|c|}{ n-3 PUFA $(n=68)$} & \multicolumn{4}{|c|}{ Control $(n=65)$} \\
\hline & Baseline & Week 24 & Difference $(\mathrm{Cl})$ & Baseline & Week 24 & Difference $(\mathrm{Cl})$ & P \\
\hline Palmitic acid (16:0) wt.\% & 12.32 & 12.40 & $0.08(-0.04-0.21)$ & 12.38 & 12.37 & $-0.00(-0.12-0.12)$ & 0.33 \\
\hline Stearic acid (18:0) wt \% & 17.36 & 17.35 & $-0.01(-0.12-0.11)$ & 17.41 & 17.30 & $-0.11(-0.24-0.01)$ & 0.20 \\
\hline Oleic acid (18:1n-9) wt.\% & 32.90 & 33.21 & $0.30(0.03 ; 0.57)$ & 32.97 & 33.44 & $0.47(0.24 ; 0.70)$ & 0.35 \\
\hline Linoleic acid (18:2n-6) wt. \% & 9.12 & 8.62 & $-0.50(-0.80 ;-0.21)$ & 9.32 & 9.21 & $-0.11(-0.26 ; 0.05)$ & 0.02 \\
\hline AA (20:4n-6) wt \% & 13.05 & 11.44 & $-1.61(-1.84 ;-1.38)$ & 13.01 & 12.80 & $-0.20(-0.37 ;-0.04)$ & $<0.01$ \\
\hline Total n-6 wt \% & 22.17 & 20.05 & $-2.12(-2.47--1.76)$ & 22.32 & 22.01 & $-0.31(-0.51--0.11)$ & $<0.01$ \\
\hline EPA wt \% & 0.60 & 1.99 & $1.40(1.24 ; 1.56)$ & 0.53 & 0.50 & $-0.03(-0.08 ; 0.02)$ & $<0.01$ \\
\hline DPA wt $\%$ & 1.58 & 2.61 & $1.03(0.86 ; 1.21)$ & 1.46 & 1.42 & $-0.04(-0.10 ; 0.02)$ & $<0.01$ \\
\hline DHA wt \% & 1.21 & 2.10 & $0.89(0.77 ; 1.00)$ & 1.14 & 1.11 & $-0.02(-0.07 ; 0.02)$ & $<0.01$ \\
\hline Total n-3 PUFA (DHA + EPA + DPA) wt \% & 3.39 & 6.71 & $3.32(2.92 ; 3.71)$ & 3.13 & 3.03 & $-0.10(-0.22 ; 0.03)$ & $<0.01$ \\
\hline
\end{tabular}

$A A$ arachidonic acid, DHA docosahexaenoic acid, EPA eicosapentaenoic acid, DPA docosapentaenoic acid, wt. \% weight percent, $P$ P for the difference between the two groups of supplement

A likely explanation for a low HRV in PsA is the presence of systemic inflammation leading to a decreased parasympathetic regulation of cardiac autonomic tone $[51,52]$. In this study, DAS66/68 and CRP were not associated with HRV or PWV. However, DAS and CRP may be a more important marker of disease activity in patients with RA [53] than in patients with PsA $[47,54,55]$.

In a small study with 20 patients with PsA, Syngle et al. observed improvement in autonomic dysfunction after treatment with different synthetic DMARDs over 12 weeks [33]. However, no treatment strategy for cardiac autonomic dysfunction in PsA has yet been demonstrated. Thus, the possible beneficial effect of n-3
PUFA found in our study may be of importance in the approach towards an improved cardiac autonomic function in PsA.

n-3 PUFA is known to have a mildly antihypertensive effect [56]. Furthermore, studies and a meta-analysis of randomized and controlled human clinical trials examining the effect of n-3 PUFA on arterial stiffness has shown a reduction in arterial stiffness after treatment with less than $4 \mathrm{~g} / \mathrm{d}$ of $\mathrm{n}-3$ PUFA in populations with hypertension, diabetes mellitus, dyslipidemia, metabolic syndrome and obesity $[57,58]$.

In our study we were unable to demonstrate any changes in BP, PWV, central BP and AIx measurements. However, in most of the previous studies arterial

Table 3 Intention to treat data with no adjustments

\begin{tabular}{|c|c|c|c|c|c|c|c|}
\hline & $n-3$ PUFA $\quad(n=68)$ & & & Control $(n=60)$ & & & \\
\hline & Baseline & Week 24 & Difference $(\mathrm{Cl})$ & Baseline & Week 24 & Difference $(\mathrm{Cl})$ & $P$ \\
\hline Heart rate, $\min ^{-1}$ & 63.83 & 63.29 & $-0.61(-1.92 ; 0.70)$ & 63.39 & 64.38 & $0.96(-0.55 ; 2.47)$ & 0.12 \\
\hline $\mathrm{RR}$, ms & 956.55 & 969.94 & $13.38(-5.06 ; 31.83)$ & 964.02 & 950.53 & $-13.48(-35 ;-8.69)$ & 0.06 \\
\hline PNN50 \% & 10.68 & 11.29 & $0.62(-3.25 ; 4.48)$ & 15.83 & 14.37 & $-1.46(-4.76 ; 1.84)$ & 0.42 \\
\hline SDNN ms & 49.37 & 48.12 & $-1.24(-8.46 ; 5.97)$ & 49.71 & 47.41 & $-2.30(-6.79 ; 2.20)$ & 0.81 \\
\hline RMSSD ms & 36.59 & 37.58 & $0.99(-7.13 ; 9.12)$ & 39.84 & 39.15 & $-0.69(-6.23 ; 4.85)$ & 0.73 \\
\hline Peripheral systolic BP mmHg & 138.20 & 134.53 & $-3.67(-6.69 ;-0.65)$ & 134.41 & 133.18 & $-1.23(-4.59 ; 2.14)$ & 0.28 \\
\hline Peripheral diastolic BP $\mathrm{mmHg}$ & 82.61 & 81.82 & $-0.79(-2.35 ; 0.77)$ & 82.36 & 80.92 & $-1.44(-3.26 ; 0.39)$ & 0.59 \\
\hline $\mathrm{PWV} \mathrm{m/s}$ & 7.80 & 7.81 & $0.01(-0.44 ; 0.46)$ & 7.40 & 7.48 & $0.08(-0.33 ; 0.49)$ & 0.82 \\
\hline Central systolic BP mmHg & 114.82 & 112.24 & $-2.58(-4.84 ;-0.32)$ & 113.29 & 111.38 & $-1.91(-4.77 ; 0.95)$ & 0.71 \\
\hline $\begin{array}{l}\text { Central diastolic BP } \\
\mathrm{mmHg}\end{array}$ & 96.04 & 93.76 & $-2.28(-4.10 ;-0.47)$ & 95.45 & 94.17 & $-1.29(-3.51 ; 0.94)$ & 0.49 \\
\hline PWA Alx & 26.42 & 27.54 & $1.12(-0.56 ; 2.80)$ & 26.97 & 25.82 & $-1.15(-2.78 ; 0.47)$ & 0.05 \\
\hline
\end{tabular}

Outcomes presented as means with $95 \%$ confidence intervals at baseline and after 24 weeks of supplement for both groups

$C I$ Confidence Interval, $P$ P for difference between the two groups of supplement, HRV Heart rate variability, PWV Pulse wave velocity, $B P$ blood pressure, $A / x$ central Augmentation Index 
Table 4 Per-protocol data with no adjustments

\begin{tabular}{|c|c|c|c|c|c|c|c|}
\hline & \multicolumn{3}{|c|}{ n-3 PUFA $(n=58)$} & \multicolumn{4}{|c|}{ Control $(n=56)$} \\
\hline & Baseline & Week 24 & Difference $(\mathrm{Cl})$ & Baseline & Week 24 & Difference $(\mathrm{Cl})$ & $P$ \\
\hline Heart rate & 63.24 & 61.73 & $-1.51(-2.89 ;-0.13)$ & 63.39 & 64.38 & $0.98(-0.54 ; 2.50)$ & 0.02 \\
\hline RR ms & 964.14 & 990.39 & $26.25(6.21 ; 46.30)$ & 964.02 & 950.53 & $-13.48(-35.66 ; 8.69)$ & 0.01 \\
\hline PNN50 \% & 11.39 & 11.65 & $0.26(-4.06 ; 4.58)$ & 15.83 & 14.37 & $-1.46(-4.76 ; 1.84)$ & 0.52 \\
\hline SDNN ms & 51.56 & 49.36 & $-2.20(-9.96 ; 5.56)$ & 49.71 & 47.41 & $-2.30(-6.79 ; 2.20)$ & 0.98 \\
\hline RMSSD ms & 39.10 & 37.85 & $-1.25(-9.41 ; 6.91)$ & 39.84 & 39.15 & $-0.69(-6.23 ; 4.85)$ & 0.91 \\
\hline Peripheral BP Systolic mmHg & 137.67 & 134.29 & $-3.38(-7.03 ; 0.26)$ & 134.41 & 133.18 & $-1.23(-4.59 ; 2.14)$ & 0.39 \\
\hline Peripheral BP Diastolic mmHg & 81.96 & 80.88 & $-1.08(-2.89 ; 0.74)$ & 82.36 & 80.92 & $-1.44(-3.26 ; 0.39)$ & 0.78 \\
\hline $\mathrm{PWV} \mathrm{m/s}$ & 7.66 & 7.61 & $-0.04(-0.51 ;-0.43)$ & 7.40 & 7.48 & $0.08(-0.33 ; 0.49)$ & 0.70 \\
\hline Central BP systolic mmHg & 114.62 & 112.17 & $-2.44(-5.17 ; 0.28)$ & 113.29 & 111.38 & $-1.91(-4.77 ; 0.95)$ & 0.79 \\
\hline Central BP diastolic mmHg & 95.50 & 93.13 & $-2.37(-4.49 ;-0.24)$ & 95.45 & 94.17 & $-1.29(-3.51 ; 0.94)$ & 0.49 \\
\hline PWA Alx & 26.42 & 27.51 & $1.12(-0.56 ; 2.80)$ & 27.02 & 25.84 & $-1.15(-2.78 ; 0.47)$ & 0.06 \\
\hline
\end{tabular}

Outcomes presented as means with $95 \%$ confidence intervals at baseline and after 24 weeks of supplement for both groups

$\mathrm{Cl}$ Confidence Interval, P P for difference between the two groups of supplement, PWV Pulse wave velocity, BP blood pressure, Alx central Augmentation Index

stiffness was not assessed with carotid-femoral PWV (regarded as "golden standard") as in our study. Supplementation with n-3-PUFA for 24 weeks might be too short a period to observe changes and modulation in the vessels measured by PWV. Patients in this study had a low mean disease activity score of $2.6(\mathrm{sd}=0.9)$ at baseline and $75 \%$ of the patients received disease-modifying antirheumatic drugs. Thus, we investigated a patient group in remission and results may not apply to patients with a more severely disease activity. In other chronic inflammatory diseases, such as RA and systemic vasculitis, PWV and AIx were increased compared to controls, but only in patients with active disease $[59,60]$.

\section{Limitation of the study}

Five minutes HRV was used to assess the function of the autonomic nervous system. This method limits the measurement of vagal predominance during nighttime.
However, other studies assessing patients with PsA have also obtained 5 min HRV with results comparable to our findings.

The difference in HRV in the intention to treat and per-protocol results might be explained by noncompliance. Compliance and adherence to the study protocol might have been improved with administration of more concentrated capsules of n-3 PUFA resulting in fewer capsules per day for the patients.

Olive oil has often been used as control oil in studies investigating the effects of fish oil in patients with arthritis but olive oil itself may have some effect on inflammation [61]. However, marine n-3 PUFA have been shown to be superior to olive oil in several studies of CVD [37] and olive oil had no anti-arrhythmic effect in previous studies $[62,63]$. The mean intake of monounsaturated fat in Denmark is $36 \mathrm{~g} / \mathrm{d}$ [64] and therefore, adding $3 \mathrm{~g} / \mathrm{d}$ of olive oil would not be expected to have

Table 5 Per-protocol analysis

\begin{tabular}{|c|c|c|c|c|}
\hline & Estimated effect $(95 \% \mathrm{Cl})$ & F statistic & $P$ & $\mathrm{~N}$ \\
\hline $\mathrm{RR} \mathrm{ms}$ & 35.26 (3.00 to 67.53$)$ & 4.70 & 0.03 & 114 \\
\hline PNN50 \% & $1.81(-3.99$ to 7.61$)$ & 0.38 & 0.54 & 114 \\
\hline SDNN ms & $-0.05(-9.49$ to 9.39$)$ & 0.00 & 0.99 & 114 \\
\hline RMSSD ms & $1.35(-9.06$ to 11.76$)$ & 0.07 & 0.80 & 114 \\
\hline Peripheral systolic BP, mmHg & $-1.48(-6.25$ to 3.29$)$ & 0.38 & 0.54 & 117 \\
\hline Peripheral diastolic BP, mmHg & $0.26(-2.45$ to 2.96$)$ & 0.03 & 0.85 & 117 \\
\hline Pulse Wave Velocity, m/s & $-0.15(-0.83$ to 0.54$)$ & 0.18 & 0.67 & 111 \\
\hline Central systolic BP mmHg & $-0.90(-5.23$ to 3.44$)$ & 0.17 & 0.68 & 117 \\
\hline Central diastolic BP mmHg & $-1.57(-4.94$ to 1.79$)$ & 0.86 & 0.36 & 117 \\
\hline PWA Alx & $2.91(0.12$ to 5.71$)$ & 4.26 & 0.04 & 117 \\
\hline
\end{tabular}

ANCOVA analysis of differences in outcomes from baseline to 24 weeks comparing n-3 PUFA to control. Analysis are controlled for age, sex, smoking, diabetes mellitus, hypertension, blood pressure, hypercholesterolemia, NSAID treatment and Disease activity scores. $\mathrm{N}$ are numbers of patients

$\mathrm{Cl}$ Confidence Interval, HRV Heart rate variability, BP Blood pressure, PWV Pulse wave velocity, Alx central Augmentation Index 
a substantial effect on the results. Also, we found no significant changes in the content in granulocytes of oleic acid and linoleic acid (main components of olive oil) in the control group after 24 weeks of supplementation (Table 2).

\section{Conclusion}

In conclusion, our study demonstrated a beneficial effect of n-3 PUFA on RR and HR in a well-characterized group of patients with PsA. The results may indicate a beneficial effect of $n-3$ PUFA on cardiac autonomic tone in patients with PsA and further large-scale studies are needed to demonstrate whether this translates into a reduction of CVD in these patients.

\section{Abbreviations}

AA: Arachidonic acid; Al: Augmentation index; Alx: Aortic augmentation index; ANCOVA: Analysis of covariance; ANOVA: One-way analysis of variance; BMI: Body mass index; BP: Blood pressure; CASPAR: Classification criteria for psoriatic arthritis; Cl: Confidence intervals; CRP: C-reactive protein; CVD: Cardiovascular disease; DAS: Disease activity score;

DHA: Docosahexaenoic acid; DMARD: Disease-modifying antirheumatic drugs; DPA: Docosapentaenoic acid; EPA: Eicosapentaenoic acid; GCP: Good clinical practice; HR: Heart rate; HRV: Heart rate variability; LA: Linoleic acid; $n-$ 3 PUFA: n-3 polyunsaturated fatty acids; NSAID: Nonsteroidal antiinflammatory drugs; PASI: Psoriatic skin area involvement; pNN50: Percentage of successive RR-interval differences > 50 ms; PsA: Psoriatic arthritis; PWV: Pulse wave velocity; RMSSD: Square root of the mean of the sum of the squares of differences between adjacent intervals; RR: Mean of all normal RR intervals during the 5 min recording; SD: Standard deviation; SDNN: Standard deviation of all normal RR intervals in the 5 min recording; SDNNindex: Mean of the standard deviation of all the normal RR intervals
\end{abstract}

\section{Acknowledgements}

We express our gratitude to the patients for participating in this study. We thank C. M. Skov, K. Holdensen, V. Mellergaard, R. B. Eschen, A. Andreasen and B. Thomsen for invaluable laboratory assistance and excellent work participating in the examinations.

\section{Funding}

The study was supported by Aalborg University Hospital Research Foundation, The Medical Research Foundation of the Northern Region, Denmark, The Danish Rheumatism Association, The Danish Psoriasis Foundation, The Aage Bang Foundation, Abbvie Foundation, Heinrich Kopps Foundation and Jacob Madsen and wife Olga Madsen Foundation.

The capsules of marine n-3 PUFA and olive oil were kindly delivered by Marine Pharma, Norway.

\section{Availability of data and materials}

The datasets generated during the current study are available in the Clinical Trials, www.clinicaltrials.gov Identifier: NCT01818804.

\section{Authors' contributions \\ SK designed and coordinated the study, collected data, performed statistical analysis and drafted and revised the manuscript. JHC, EBS, and AS participated in the study design and data interpretation and were involved in critically revising the manuscript for important intellectual content. CR participated in the study design and coordination and helped to revise the manuscript. EL helped collecting data and revising the manuscript. MBJ contributed to the study design and helped with the statistical analysis. All authors read and approved the final manuscript.}

\section{Competing interests}

The authors declare that they have no competing interests.

\section{Consent for publication}

Not applicable.

\section{Ethics approval and consent to participate}

All participants gave their written informed consent and the regional ethics committee of Northern Region DK, approved the study (reference number N20120076).

\section{Author details}

'Department of Rheumatology, Aalborg University Hospital, Reberbansgade 14, 9000 Aalborg, Denmark. ${ }^{2}$ Department of Cardiology, Aalborg University Hospital, Department of Clinical Medicine, Aalborg University, 9000 Aalborg, Denmark. ${ }^{3}$ Department of Rheumatology, North Denmark Regional Hospital, 9800 Hjørring, Denmark. ${ }^{4}$ Department of Cardiology and Unit of Clinical Biostatistics and Bioinformatics, Aalborg University Hospital, 9000 Aalborg, Denmark. ${ }^{5}$ Department of Nephrology, Aalborg University Hospital, Department of Clinical Medicine, Aalborg University, 9000 Aalborg, Denmark.

Received: 19 August 2016 Accepted: 29 November 2016

Published online: 12 December 2016

\section{References}

1. Gelfand JM, Gladman DD, Mease PJ, Smith N, Margolis DJ, Nijsten T, Stern RS, Feldman SR, Rolstad T. Epidemiology of psoriatic arthritis in the population of the United States. J Am Acad Dermatol. 2005;53(4):573-7.

2. Shbeeb M, Uramoto KM, Gibson LE, O'Fallon WM, Gabriel SE. The epidemiology of psoriatic arthritis in Olmsted County, Minnesota, USA, 1982-1991. J Rheumatol. 2000;27(5):1247-50.

3. Wilson FC, Icen M, Crowson CS, McEvoy MT, Gabriel SE, Kremers HM. Incidence and clinical predictors of psoriatic arthritis in patients with psoriasis: a population-based study. Arthritis Rheum. 2009;61(2):233-9.

4. Han C, Robinson Jr DW, Hackett MV, Paramore LC, Fraeman KH, Bala MV. Cardiovascular disease and risk factors in patients with rheumatoid arthritis, psoriatic arthritis, and ankylosing spondylitis. J Rheumatol. 2006;33(11):2167-72.

5. Ahlehoff $\mathrm{O}$, Gislason $\mathrm{GH}$, Charlot M, Jorgensen $\mathrm{CH}$, Lindhardsen J, Olesen JB, Abildstrom SZ, Skov L, Torp-Pedersen C, Hansen PR. Psoriasis is associated with clinically significant cardiovascular risk: a Danish nationwide cohort study. J Intern Med. 2011;270(2):147-57.

6. Gladman DD, Ang M, Su L, Tom BD, Schentag CT, Farewell VT. Cardiovascular morbidity in psoriatic arthritis. Ann Rheum Dis. 2009;68(7):1131-5.

7. Kimhi O, Caspi D, Bornstein NM, Maharshak N, Gur A, Arbel Y, Comaneshter D, Paran D, Wigler I, Levartovsky D, Berliner S, Elkayam O. Prevalence and risk factors of atherosclerosis in patients with psoriatic arthritis. Semin Arthritis Rheum. 2007;36(4):203-9.

8. Bruchfeld A, Goldstein RS, Chavan S, Patel NB, Rosas-Ballina M, Kohn N, Qureshi AR, Tracey KJ. Whole blood cytokine attenuation by cholinergic agonists ex vivo and relationship to vagus nerve activity in rheumatoid arthritis. J Intern Med. 2010;268(1):94-101.

9. Huston JM, Tracey KJ. The pulse of inflammation: heart rate variability, the cholinergic anti-inflammatory pathway and implications for therapy. J Intern Med. 2011;269(1):45-53.

10. Stein PK, Kleiger RE. Insights from the study of heart rate variability. Annu Rev Med. 1999:50:249-61.

11. Tsuji H, Larson MG, Venditti Jr FJ, Manders ES, Evans JC, Feldman CL, Levy D. Impact of reduced heart rate variability on risk for cardiac events. The Framingham Heart Study. Circulation. 1996;94(11):2850-5.

12. La Rovere MT, Bigger JT, Marcus FI, Mortara A, Schwartz PJ. Baroreflex sensitivity and heart-rate variability in prediction of total cardiac mortality after myocardial infarction. ATRAMI. Lancet. 1998;351(9101):478-84.

13. Schwartz PJ. The autonomic nervous system and sudden death. Eur Hear J. 1998;19(Suppl F):F72-80.

14. Barron HV, Lesh MD. Autonomic nervous system and sudden cardiac death J Am Coll Cardiol. 1996;27(5):1053-60.

15. Airaksinen KEJ. Autonomic mechanisms and sudden death after abrupt coronary occlusion. Ann Med. 1999;31(4):240-5.

16. Laurent S, Boutouyrie P, Asmar R, Gautier I, Laloux B, Guize L, Ducimetiere P, Benetos A. Aortic stiffness is an independent predictor of all-cause and cardiovascular mortality in hypertensive patients. Hypertension. 2001;37(5): $1236-41$.

17. Willum-Hansen T, Staessen JA, Torp-Pedersen C, Rasmussen S, Thijs L, Ibsen $\mathrm{H}$, Jeppesen J. Prognostic value of aortic pulse wave velocity as index of arterial stiffness in the general population. Circulation. 2006;113(5):664-70.

18. Gelfand JM, Neimann AL, Shin DB, Wang X, Margolis DJ, Troxel AB. Risk of myocardial infarction in patients with psoriasis. JAMA. 2006;296(14):1735-41. 
19. Gisondi P, Fantin F, Del Giglio M, Valbusa F, Marino F, Zamboni M, Girolomoni G. Chronic plaque psoriasis is associated with increased arteria stiffness. Dermatology. 2009;218(2):110-3.

20. Costa L, Caso F, D'Elia L, Atteno M, Peluso R, Del Puente A, Strazzullo P, Scarpa R. Psoriatic arthritis is associated with increased arterial stiffness in the absence of known cardiovascular risk factors: A case control study. Clin Rheumatol. 2012;31(4):711-5.

21. De Caterina R. N-3 Fatty Acids in Cardiovascular Disease. N Engl J Med. 2011;364(25):2439-50.

22. Calder PC. Marine omega-3 fatty acids and inflammatory processes: Effects, mechanisms and clinical relevance. Biochim Biophys Acta. 2014;1851(4):469-84.

23. Christensen JH, Gustenhoff P, Korup E, Aarøe J, Toft E, Møller J, Rasmussen K, Dyerberg J, Schmidt EB. Effect of fish oil on heart rate variability in survivors of myocardial infarction: a double blind randomised controlled trial. BMJ. 1996:312(7032):677-8.

24. Saravanan P, Davidson NC, Schmidt EB, Calder PC. Cardiovascular effects of marine omega-3 fatty acids. Lancet. 2010;376(9740):540-50.

25. Taylor W, Gladman D, Helliwell P, Marchesoni A, Mease P, Mielants H. Classification criteria for psoriatic arthritis: Development of new criteria from a large international study. Arthritis Rheum. 2006;54(8):2665-73.

26. Maksymowych WP, Mallon C, Morrow S, Shojania K, Olszynski WP, Wong RL, Sampalis J, Conner-Spady B. Development and validation of the Spondyloarthritis Research Consortium of Canada (SPARCC) Enthesitis Index. Ann Rheum Dis. 2009;68(6):948-53.

27. Asmar R, Benetos A, Topouchian J, Laurent P, Pannier B, Brisac AM, Target $R$, Levy B. Assessment of arterial distensibility by automatic pulse wave velocity measurement. Validation and clinical application studies. Hypertension. 1995;26(3):485-90.

28. Laurent S, Cockcroft J, Van Bortel L, Boutouyrie P, Giannattasio C, Hayoz D, Pannier B, Vlachopoulos C, Wilkinson I, Struijker-Boudier H, A. European Network for Non-invasive Investigation of Large. Expert consensus document on arterial stiffness: methodological issues and clinical applications. Eur Hear J. 2006;27(21):2588-605.

29. Van Bortel LM, Laurent S, Boutouyrie P, Chowienczyk P, Cruickshank JK, De Backer T, Filipovsky J, Huybrechts S, Mattace-Raso FUS, Protogerou AD, Schillaci G, Segers P, Vermeersch S, Weber T. Expert consensus document on the measurement of aortic stiffness in daily practice using carotidfemoral pulse wave velocity. J Hypertens. 2012;30(3):445-8.

30. Dzeko M, Peters CD, Kjaergaard KD, Jensen JD, Jespersen B. Aortic pulse wave velocity results depend on which carotid artery is used for the measurements. J Hypertens. 2013;31(1):117-22.

31. Chen C-H, Nevo E, Fetics B, Pak PH, Yin FCP, Maughan WL, Kass DA. Estimation of Central Aortic Pressure Waveform by Mathematical Transformation of Radial Tonometry Pressure : Validation of Generalized Transfer Function. Circulation. 1997:95(7):1827-36.

32. Pauca AL, O'Rourke MF, Kon ND. Prospective evaluation of a method for estimating ascending aortic pressure from the radial artery pressure waveform. Hypertension. 2001;38(4):932-7.

33. Syngle A, Verma I, Krishan P, Garg N, Syngle V. Disease-modifying antirheumatic drugs improve autonomic neuropathy in arthritis: DIANA study. Clin Rheumatol. 2014;34(7):1233-41.

34. Janse van Rensburg DC, Ker JA, Grant CC, Fletcher L. Effect of exercise on cardiac autonomic function in females with rheumatoid arthritis. Clin Rheumatol. 2012;31(8):1155-62.

35. Task Force of the European Society of Cardiology; t. N. A. S. Electrophysiology. Heart Rate Variability: Standards of Measurement, Physiological Interpretation, and Clinical Use. Circulation. 1996;93(5):1043-65.

36. Kleiger RE, Miller JP, Bigger JT, Moss AJ. Decreased heart rate variability and its association with increased mortality after acute myocardial infarction. Am J Cardiol. 1987;59(4):256-62.

37. La Rovere MT, Christensen JH. The autonomic nervous system and cardiovascular disease: role of n-3 PUFAs. Vascul Pharmacol. 2015;71:1-10.

38. Christensen JH. Omega-3 polyunsaturated Fatty acids and heart rate variability. Front Physiol. 2011;2:84.

39. Mozaffarian D, Geelen A, Brouwer IA, Geleijnse JM, Zock PL, Katan MB. Effect of fish oil on heart rate in humans: a meta-analysis of randomized controlled trials. Circulation. 2005;112(13):1945-52.

40. Dallongeville J, Yarnell J, Ducimetière $P$, Arveiler D, Ferfières J, Montaye M, Luc G, Evans A, Bingham A, Hass B, Ruidavets JB, Amouyel P. Fish consumption is associated with lower heart rates. Circulation. 2003; 108(7):820-5.
41. Palatini P, Julius S. Elevated Heart Rate: A Major Risk Factor for Cardiovascular Disease. Clin Exp Hypertens. 2004:26:637-44.

42. La Rovere MT, Staszewsky L, Barlera S, Maestri R, Mezzani A, Midi P, Marchioli R, Maggioni AP, Tognoni G, Tavazzi L, Latini R. n-3PUFA and Holter-derived autonomic variables in patients with heart failure: data from the Gruppo Italiano per lo Studio della Sopravvivenza nell'Insufficienza Cardiaca (GISSI-HF) Holter substudy. Heart Rhythm. 2013;10(2):226-32.

43. Billman GE. Heart Rate Variability? A Historical Perspective. Front Physiol. 2011:2(November):1-13

44. Christensen $J \mathrm{H}$, Schmidt EB. Autonomic nervous system, heart rate variability and n-3 fatty acids. J Cardiovasc Med. 2007:8 Suppl 1:S19-22.

45. Grimsgaard S, Bønaa KH, Hansen JB, Myhre ES. Effects of highly purified eicosapentaenoic acid and docosahexaenoic acid on hemodynamics in humans. Am J Clin Nutr. 1998;68(1):52-9.

46. Innis SM. Dietary (n-3) fatty acids and brain development. J Nutr. 2007; 137(4):855-9.

47. Helliwell PS. Assessment of disease activity in psoriatic arthritis. 2015;33(5 Suppl 93):S44-7.

48. Stojanovich L. Autonomic dysfunction in autoimmune rheumatic disease. Autoimmun Rev. 2009:8(7):569-72.

49. Gaydukova I, Rebrov A, Nikitina N, Poddubnyy D. Decreased heart rate variability in patients with psoriatic arthritis. Clin Rheumatol. 2012;31(9): 1377-81.

50. Proietti I, Raimondi G, Skroza N, Pampena R, Bernardini N, La Viola G, Nicolucci F, Tolino E, Zuber S, Scordamaglia B, Balduzzi V, Soccodato V, Potenza C. Cardiovascular Risk in Psoriatic Patients Detected by Heart Rate Variability (HRV) Analysis. Drug Dev Res. 2014;75:S81-4.

51. Madsen T, Christensen JH, Toft E, Schmidt EB. C-reactive protein is associated with heart rate variability. Ann Noninvasive Electrocardiol. 2007;12(3):216-22.

52. Sloan RP, Mccreath H, Tracey KJ, Sidney S, Liu K, Seeman T. RR Interval Variability Is Inversely Related to Inflammatory Markers : The CARDIA Study. Mol Med. 2007;13(3-4):178-84.

53. Spiegel TM, King W, Weiner SR, Paulus HE. Measuring disease activity: comparison of joint tenderness, swelling, and ultrasonography in rheumatoid arthritis. Arthritis Rheum. 1987:30:1283-8.

54. Daunt AO, Cox NL, Robertson JC, Cawley MI. Indices of disease activity in psoriatic arthritis. J R Soc Med. 1987:80:556-8.

55. Coates L. Outcome Measures in Psoriatic Arthritis. Rheum Dis Clin North Am. 2015:41(4):699-710.

56. Morris MC, Sacks F, Rosner B. Does fish oil lower blood pressure? A metaanalysis of controlled trials. Circulation. 1993;88(2):523-33.

57. Pase MP, Grima NA, Sarris J. Do long-chain n-3 fatty acids reduce arterial stiffness? A meta-analysis of randomised controlled trials. Br J Nutr. 2011; 106(7):974-80

58. Monahan KD, Feehan RP, Blaha C, McLaughlin DJ. Effect of omega-3 polyunsaturated fatty acid supplementation on central arterial stiffness and arterial wave reflections in young and older healthy adults. Physiol Rep. 2015:3(6):e12438.

59. Booth AD, Wallace S, McEniery CM, Brown J, Jayne DR, Wilkinson IB. Inflammation and arterial stiffness in systemic vasculitis: a model of vascular inflammation. Arthritis Rheum. 2004;50(2):581-8.

60. Provan SA, Semb AG, Hisdal J, Stranden E, Agewall S, Dagfinrud H, Angel K, Atar D, Kvien TK. Remission is the goal for cardiovascular risk management in patients with rheumatoid arthritis: a cross-sectional comparative study. Ann Rheum Dis. 2011:70(5):812-7.

61. Kremer JM, Lawrence DA, Jubiz W, DiGiacomo R, Rynes R, Bartholomew LE, Sherman M. Dietary fish oil and olive oil supplementation in patients with rheumatoid arthritis. Clinical and immunologic effects. Arthritis Rheum. 1990:33(6):810-20.

62. Kirkegaard E, Svensson M, Strandhave C, Schmidt EB, Jorgensen KA, Christensen JH. Marine n-3 fatty acids, atrial fibrillation and QT interval in haemodialysis patients. Br J Nutr. 2012;107:903-9.

63. McLennan PL. Relative effects of dietary saturated, monounsaturated, and polyunsaturated fatty acids on cardiac arrhythmias in rats. Am J Clin Nutr. 1993;57(2):207-12

64. Knudsen VK, Matthiessen J, Biltoft-Jensen A, Sorensen MR, Groth MV, Trolle E, Christensen T, Fagt S. Identifying dietary patterns and associated healthrelated lifestyle factors in the adult Danish population. Eur J Clin Nutr. 2014; 68(6):736-40. 